\title{
Energy Imbalance Between the Earth and Space Controls the Climate
}

\author{
Habibullo Abdussamatov \\ Pulkovo Observatory of the RAS, St. Petersburg, Russia
}

\section{Email address:}

abduss@gaoran.ru

\section{To cite this article:}

Habibullo Abdussamatov. Energy Imbalance Between the Earth and Space Controls the Climate. Earth Sciences. Vol. 9, No. 4, 2020, pp. 117-125. doi: 10.11648/j.earth.20200904.11

Received: April 26, 2020; Accepted: June 20, 2020; Published: July 30, 2020

\begin{abstract}
The climate system depends at an extremely complex set of long-term (about 30 years or more) physical processes in the ocean-land-atmosphere systems, which, in turn, are influenced mainly quasi-bicentennial variations of the total solar irradiance (TSI). The TSI decline phase started around 1990. The onset of the Grand minimum phase of the TSI quasibicentennial cycle of the Maunder type is predicted in the 27th \pm 1 cycle in $2043 \pm 11$. Long period of deficiency of absorbed solar energy since about 1990 was not compensated by a decrease in the Earth's thermal energy emitted into space, since it does not have time to cool down due to thermal inertia, and it continues to radiate heat in the same high volumes. Solar cooling has started. As a result, the Earth has, and will continue to have, a long negative energy balance, which will ensure a slight decrease in temperature. However, this slight decrease in temperature is extremely important as a trigger mechanism for the subsequent chain effects of secondary causal effects of feedback that will greatly enhance the cooling. This will certainly lead to the onset of a phase of deep cooling of the climate approximately in the year $2070 \pm 11$. The temperature is always cooler (with some time delay) in the during long-term periods of TSI decline phase of the TSI quasi-bicentennial cycle and warmer in the during periods of its growth phase. The climate sensitivity to the atmospheric carbon dioxide abundance, due to the significant overlap of the spectral absorption bands of the water vapor and carbon dioxide, decreases as a result of a significant increase in the concentration of water vapor directly in the near-surface layer of the troposphere during warming. The impact of a long-term cloud coverage growth on climate change is also virtually nonexistent.
\end{abstract}

Keywords: Climate, Energy Balance, Solar Irradiance, Cooling, Grand Solar Minimum, Thermal Energy, Feedback Effects, Little Ice Age, Climate Sensitivity

\section{Introduction}

An extremely complex and non-linear climate system depends on the variation of a complex the numerous longterm (about 30 years or more) geophysical processes in ocean-land-atmosphere systems, which, in turn, are influenced by many different factors. Among them, the main and predominant factor is the quasi-bicentennial change of TSI, accompanied by subsequent significant manifestations of geophysical and climatic processes. One of the most important features of our star is the existence of cyclically alternating periods of growth and decrease in TSI and the number of different manifestations of its sunspot-forming activity. The Sun radiates stronger or weaker, subject to strict small 11-year (11 \pm 3 years) and grand quasi-bicentennial (200 \pm 70 years) cycles [1-8]. All these changes in the physical regime of the Sun will certainly be reflected in the states of near-Earth outer space and the planet Earth, as well as the entire Solar System (SS). The Sun, being the main source of energy for the Earth, controls the climate system, and even the smallest long-term changes in TSI can have serious consequences for the climate [9]. The quasi-bicentennial variation of the TSI and the changes associated with its longterm impact on the surface and in the Earth's atmosphere (changes in the Earth's Bond albedo and the concentration of the main greenhouse gas $(\mathrm{GHG})$ - water vapor in the first place) is one of the main sources of thermal instability and disruption Earth energy balance. The energy balance of a system of the surface - atmosphere is always turning out to be disturbed, and we have either a positive or negative balance due to 11-year and quasi-bicentennial variations TSI, and the effects of thermal inertia of the World Ocean 
(hereinafter referred to as the Ocean). Unbalanced state in the arrival and consumption of incoming solar energy in the energy balance of the Earth at the outer boundary of its atmosphere is the main state of the climate system.

The study of long-term variations in magnitude of the deviations planet's energy balance from the equilibrium state under the influence of variations TSI, and the energies of the reflected by planet fraction of TSI going back into space (determined by the value Bond albedo), and the Earth's own thermal radiation, underlying climate change, is extremely an important fundamental problem in a deep understanding of the physical mechanisms of climate change and determining the laws of its formation. It is a unique tool for understanding

$$
E=0.25\left[\left(S_{\odot}+\Delta S_{\odot}\right)-\left(A_{\mathrm{BE}}+\Delta A_{\mathrm{BE}}\right)\left(S_{\odot}+\Delta S_{\odot}\right)\right]-\varepsilon \sigma\left(T_{p}+\Delta T_{p}\right)^{4},
$$

where, $S_{\odot}$ is TSI; $\Delta S_{\odot}$ is the TSI increment; $A_{\mathrm{BE}}$ is the Earth's global albedo (Bond albedo); $\Delta A_{\mathrm{BE}}$ is the increment of the Bond albedo; $\varepsilon$ is the irradiating capacity of the surface-atmosphere system; $\sigma$ is the Stefan-Boltzmann constant; $T_{p}$ is the planetary thermodynamic temperature; $\Delta T_{p}$ is the increment of the planetary thermodynamic temperature.

\section{The Relationship of the Small 11-Years and Grand Quasi-bicentennial Cycles of the TSI Variations}

The grand quasi-bicentennial component of the TSI variation consistently decreases at an accelerating (currently) rate from the 21 st cycle to the 22nd, 23rd and 24th 11-year cycles. The maximum average cyclical value TSI is observed in the 22nd cycle, which accounted in the maximum of the the physical mechanisms of global climate change and the physics of solar-terrestrial relations. Such study will allow establish the physical causes of a climate change and, therefore, will develop reliable methods for predicting its future changes. Quasi-bicentennial cyclical climate change on Earth is a natural reflection (with some time delay) of the corresponding variation of TSI. The only and most reliable way to accurately predict the depth and time of the beginning of the deep cooling phase is to study the long-term variations of the most effective global climate parameter, namely the deviation of the average annual energy balance of the Earth $(E)$ from the equilibrium state $[3,4,10]$ :

quasi-bicentennial cycle, was $1365.99 \pm 0.02 \mathrm{~W} / \mathrm{m}^{2}[2,3,4,7$, 10]. As regards the its average cyclic value in the $23 \mathrm{rd}$ cycle has already decreased on $\sim 0.1 \mathrm{~W} / \mathrm{m}^{2}$ than in the $22 \mathrm{nd}$ cycle (Figure 1). The total rate decline in TSI from cycle to cycle is currently accelerating and will reach maximum its acceleration in the next solar cycle. The observed consistent downward trend decline of TSI in four consecutive cycles suggests that such a decrease bring backs to a similar decrease during the Maunder minimum in 1645-1715. With such a decrease, the expected TSI in the minima between cycles 24 and 25, 25 and 26 can be approximately $1364.5 \pm 1.0 \mathrm{~W} / \mathrm{m}^{2}, 1363.0 \pm 2.0 \mathrm{~W} / \mathrm{m}^{2}$, respectively (with gradually decreasing of an accuracy) in accordance with the reconstructed TSI data by Shapiro et al. [5] and Egorova et al. [6] for the period from the Maunder minimum to the present day. TSI will can reach the minimum depth $1362.5 \pm 3.0 \mathrm{~W} / \mathrm{m}^{2}$ in the minima between cycles 26 and 27,27 and 28 .

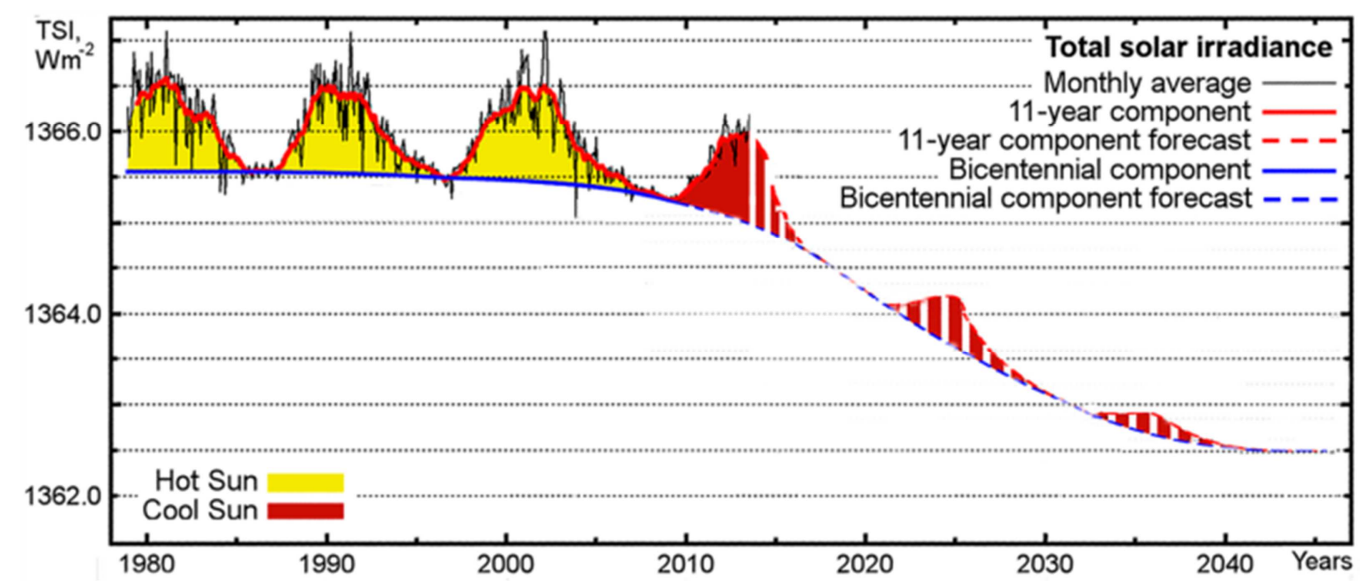

Figure 1. TSI cyclic variations [7] and our forecast of its variations to cycles 25-27 until 2045 in accordance with the reconstructed TSI data by Shapiro et al. [5] and Egorova et al. [6] for the period from the Maunder minimum to the present day (the hot Sun is marked by yellow and the cool Sun is marked by red).

We will be experiencing a period of unusually weak and cool solar cycles in the future. The duration of the small 11year cycles of TSI variations as a whole depends on the phase of the grand quasi-bicentennial cycle and sequentially increases from the growth phase to the phase's maximum and decline of the quasi-bicentennial cycle. In the decline phase of the quasi-bicentennial cycle, the duration of the 11-year cycle is $11.7 \pm 1.0$ years [11]. Such a dependence as a whole makes it possible to predict the duration of two subsequent (from the 25th to the 26th) TSI cycles, analogous to the two previous 23 and 24 cycles, which are formed during the recession of the current quasi-bicentennial cycle. Therefore, it is possible to predict the onset of the onset of the 25th

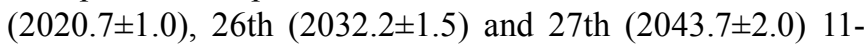


year cycles of the Sun. Grand quasi-bicentennial cycles with TSI fluctuations up to $\sim 4 \mathrm{~W} / \mathrm{m}^{2}$ play a dominant role in managing and determining the patterns of development of small daughter 11-year cycles with TSI fluctuations up to 1 $\mathrm{W} / \mathrm{m}^{2}$ in the maximum of the quasi-bicentennial cycle $[2,3$, $4,11]$. As a result of studying the relative powers of all 24 cycles and their duration, it was established that with a decrease in the energy power of the 11-year cycle, duration increases and vice versa [12].

\section{The Multiple Self-amplification of the Climate Change Thanks to the Long Chain of Subsequent Causal Feedback Effects}

The ratio of the relative contributions of the TSI increments and the Earth's Bond albedo to planetary energy balance and temperature increment is defined as $[4,10]$

$$
\frac{\Delta S_{\odot}}{S_{\odot}}=\frac{\Delta A_{B E}}{1-A_{B E}-\Delta A_{B E}} .
$$

Therefore, the Earth's Bond albedo, along with TSI, is a particularly important physical parameter in the energy balance of the planet. Being a kind of amplifier of variations in the thermal regime of our planet, it significantly increases the amplitude of corresponding quasi-bicentennial temperature changes and in particular when cooling, leads to decrease the concentration of water vapor and other GHGs in the atmosphere in accordance with relation of the ClausiusClapeyron and the law Henry. This in turn will greatly increase cooling. A long series of such cycles leads to additional cooling until the onset of the next Little Ice Age. A long chain of cycles of secondary casual feedback effects will multiply enhance the further change in warming (cooling) due to repeated repetitions of such effects, even if the TSI remains subsequently unchanged for a certain period of time. The climatic effect of feedback effects depends on the duration of the phases of the quasi-bicentennial solar cycle and can multiply enhance cooling (warming) caused by the direct influence of the corresponding long-term change in TSI. Thus, the insignificant initial temperature change caused by the direct impact of the quasi-bicentennial variation of TSI will then always additionally multiple self-strengthen significantly due to the effect of a sequential long chain of secondary climatic mechanisms of feedback effects. Indeed very slight decrease in the temperature caused by very insignificant variations in TSI (the direct effect of changes in the shape of Earth's orbit) thanks to additional to many times self-amplification of the secondary feedback effects over a period of tens thousands years leads to Grand glacial cycles with a temperature drop of about $10^{\circ} \mathrm{C}$ with a period of about 100,000 years [9].

Studying the natural variability of the climate system in the distant past allows us to evaluate the role of human civilization in climate change on the planet and to better understand possible climate changes in the future. Precise information on relative temporal changes in the temperature and the atmospheric concentration of carbon dioxide can assist in refining our understanding of the physical processes involved in this coupling. According to the ice core data drilled from a depth of over $3768 \mathrm{~m}$ near the Vostok site, Antarctica during the glacial/interglacial cycles, a rise in concentrations of greenhouse gases has begun every time after warming begins and ended after the warming was replaced by cooling (Figure 2). It is worth emphasizing that the temperature starts to decrease, after reaching its highest values in the glacial/interglacial cycles, despite the fact that the concentration of greenhouse gases continues to grow [13, 14, $15,16]$. The peaks of the carbon dioxide concentration have never preceded the warming, but on the contrary always took place $800 \pm 400$ years after it, being its consequence, i.e., they have always been a natural consequence of the temperature increase caused by long-term growth of the incoming average annual solar energy (Figure 2). According to Henry's law, warm water absorbs less gas because the solubility of a gas in a liquid is directly proportional to the partial pressure of the gas above the liquid, and hence more carbon dioxide remains in the atmosphere. So, the analysis of ice cores shows that higher or lower levels of carbon dioxide concentration are observed after warming or cooling, respectively, i.e., considerable changes in the atmospheric concentration of carbon dioxide are always determined by the corresponding temperature fluctuations of active layers Ocean.

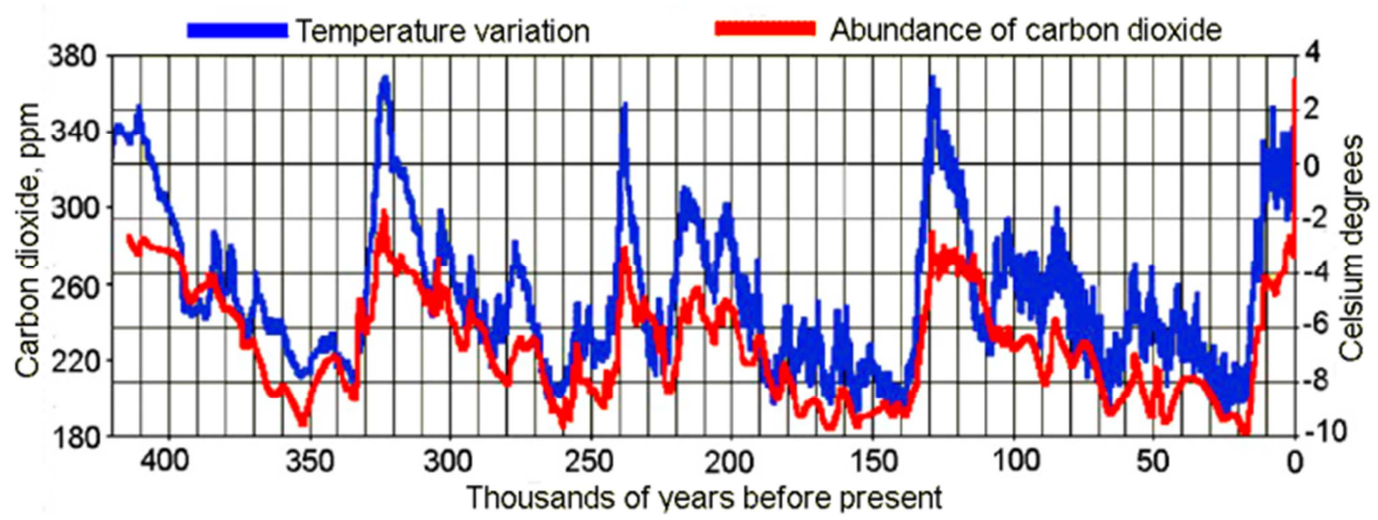

Figure 2. Variations in the Earth's climate and volumetric concentrations of carbon dioxide for a period of 420,000 years (according to the ice core data near Vostok site, Antarctica) $[13,14,16]$. 
Thus, long-term an average annual energy imbalance between the Earth and space in the outer layers of the atmosphere together with the significant subsequent influence of a long chain of feedback effects, it is fundamental to climate and its changes. Moreover, it should be emphasized that in the climate system it is also necessary to take into account the role of natural intra-systemic own short-term temperature fluctuations up to $\pm 0.2^{\circ} \mathrm{C}$, caused by change of the regional oceanic and atmospheric currents, cloudiness, snow and ice cover surface areas, etc. caused by, first of all, the uneven distribution of land on the surface of the Northern and Southern Hemispheres: $\sim 39 \%$ and $\sim 19 \%$, respectively. For example (one of many aspects), it is snowing simultaneously on a large area of the Northern and Southern Hemispheres. In the Northern Hemisphere, snow covers the land surface and leads to an increase in Bond albedo, but in the Southern Hemisphere it will melt in oceans water and the Bond albedo practically does not change.

In so doing the average annual energy of the Earth's own thermal radiation into space, due to the thermal inertia of the Ocean, always the time lag $30 \pm 10$ years from the energy of absorbed solar radiation since the planet's thermal inertia constant

$$
t=0.095(1+0.42 l) \text { years, }
$$

where $l$ is the depth of the active layer of the Ocean, which is equal to 500-1000 $\mathrm{m}$ under the current conditions [17, 29].

\section{Decrease Energy of the Grand Quasi-bicentennial Cycle TSI and Climate Cooling}

In the twentieth century in the growth phase of grand quasi-bicentennial cycle TSI the smalls 11-year cycles had a natural shorter duration, and the maximum height and the average cyclic absolute energy power gradually increased [4, 12]. As a result, for about 70 years, the Earth received more solar energy, and thanks to the thermal inertia of the Ocean, it radiated less energy into space, since it heated with a large delay (Figure 3). Consequently, during the TSI growth phase of the quasi-bicentennial cycle by our planet absorbed more energy than it emitted into space, and its energy balance remained positive for a long time $(E>0)$. As a result of receiving such by prolonged absorption and accumulation by the Ocean additional significant energy during the entire growth of the quasi-bicentennial cycle in the twentieth century, the planet was gradually heated. What is more, additional significant contribution of subsequent secondary causal feedback effects should be taken into account which caused additional global warming. And vice versa, the longterm deficit of TSI in the decay phase of the quasibicentennial cycle and, therefore, the almost proportional decrease in its share absorbed by the Earth since about 1990 was not compensated by a corresponding decrease of an average annual energy emitted back into space by it. This is because it could not cool down properly, due to the thermal inertia of the Ocean, which continues to radiate energy into the space in previous high volumes than it obtains it from there. As a result, in the phase of decline of the quasibicentennial cycle - for about 30 years, the Earth's energy budget (at present) remains negative $(E<0)$. Such a gradual expenditure of surplus (additional) solar energy accumulated in the Ocean during almost the entire 20th century led to begin a start trend gradual cooling of the planet due to the solar cooling has already begun. The energy balance of the Earth will remain negative $(E<0)$ and in subsequent 25-28th solar cycles, as the Sun moves to Grand and deep TSI minimum of the quasi-bicentennial cycle. Compensated with a large delay, determined by the thermal inertia of the Ocean, the solar energy absorbed by the Earth always forms an excess or deficit of the average annual energy balance.

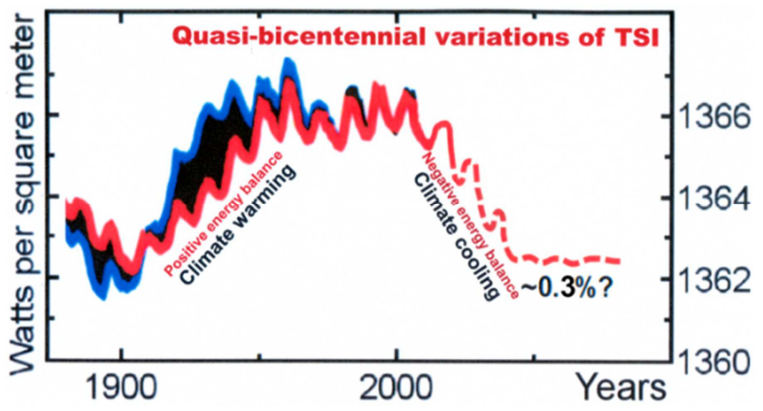

Figure 3. Quasi-bicentennial variations of TSI (data are taken from [5, 6, 7]) and our prognosis their subsequent changes until the end of the 21st century (dashed lines) and variations of the energy balance of the Earth and climate.

Always in the period $\sim 35-65$ years of the decline phase of the quasi-bicentennial cycle TSI the Earth emits more energy into space than receives and its the average annual energy balance is negative $(E<0)$, because it had not time to cool down accordingly due to the thermal inertia of the Ocean [2, $3,18]$. As a result of a long-term deficit of energy the Earth gradually will cool down insignificantly. Then the subsequent multiple influences of secondary causal chain feedback effects which cause a significant increase in the magnitude of the Earth's Bond albedo, decrease atmospheric abundance of the main GHG - water vapor and other GHG and as well as expansion of the atmospheric transparency window which reduces the temperature up to several times to comparison with the direct impact of the TSI. At the phase grow the reverse is true $(E>0)$. The average annual energy imbalance $E \neq 0$ between the Earth and space (due to 11- and 200-year variations of the TSI and thermal inertia) is the natural state of the Earth's climate system and fluctuates around its quasi-bicentennial equilibrium state. The value of difference between the maximum and minimum of the 11year smoothed cyclic variations of TSI at the maximum of the quasi-bicentennial cycle was approximately $1.0 \mathrm{~W} / \mathrm{m}^{2}$ $(\sim 0.07 \%)$ and has been gradually decreasing since approximately 1990 , since began of the decline phase of the TSI of the quasi-bicentennial cycle. The value of an average cyclic TSI in the $23 \mathrm{rd} 11$-year solar cycle became $\approx 0.1 \mathrm{~W} / \mathrm{m}^{2}$ 
less than in the 22nd cycle. The height of the maximum TSI level in the 24 th cycle decreased by some $0.5 \mathrm{~W} / \mathrm{m}^{2}$ relative to the maximum level of the 23 rd cycle (Figure 1 ). The solar cooling of the Earth has already begun.

The TSI value is currently the lowest over the past more than 75 years. The study of TSI variations during 21-24 consecutive 11- year cycles and its decrease since about 1990 made it possible to predict the onset of the phase of the Grand minimum of the Maunder type quasi-bicentennial cycle at the beginning of the 27 th \pm 1 cycle in $2043 \pm 11$ lasting 35-65 years. The total energy of solar radiation in each subsequent cycle will gradually decrease, reaching a minimum level of $2043 \pm 11$. This together with by active impact of the feedback effects will certainly lead to the onset of the deep cooling phase of the new Little Ice Age approximately in the year $2070 \pm 11$, when the average temperature across the globe will decrease by approximately $1.3^{\circ} \mathrm{C}$ (Figure 4). The deep cooling phase will last approximately 3-6 solar cycles (35-65 years), after which at the beginning of the XXII century the next quasi-bicentennial warming - cooling cycle will begin on the Earth. There are at least two more 11-year cycles of further decline in TSI, until a sufficiently deep minimum of the Maunder type is reached. As a result, over the next decades, acceleration of changes in the global characteristics of the climate system in the opposite direction is expected. The long-term energy imbalance between the Earth and space due to the decreased only TSI in decay phase quasi-bicentennial cycle by approximately $4.0 \mathrm{~W} / \mathrm{m}^{2}(\sim 0.3 \%)[5,6]$ (excluding the other contributions and at immutability of Bond albedo $\Delta A_{\mathrm{BE}}=0$ ) will provide a steady slight cooling of the climate on about $0.2 \mathrm{~K}$. However, even such a slight decrease in temperature during the TSI long decline phase of the quasi-bicentennial cycle is extremely important as a trigger mechanism for subsequent multiple exposures of a long chain of secondary causal feedback effects. They in decay phase TSI magnifies drop a several times in decrease temperature, caused by direct impact of long TSI deficiency, thanks to:

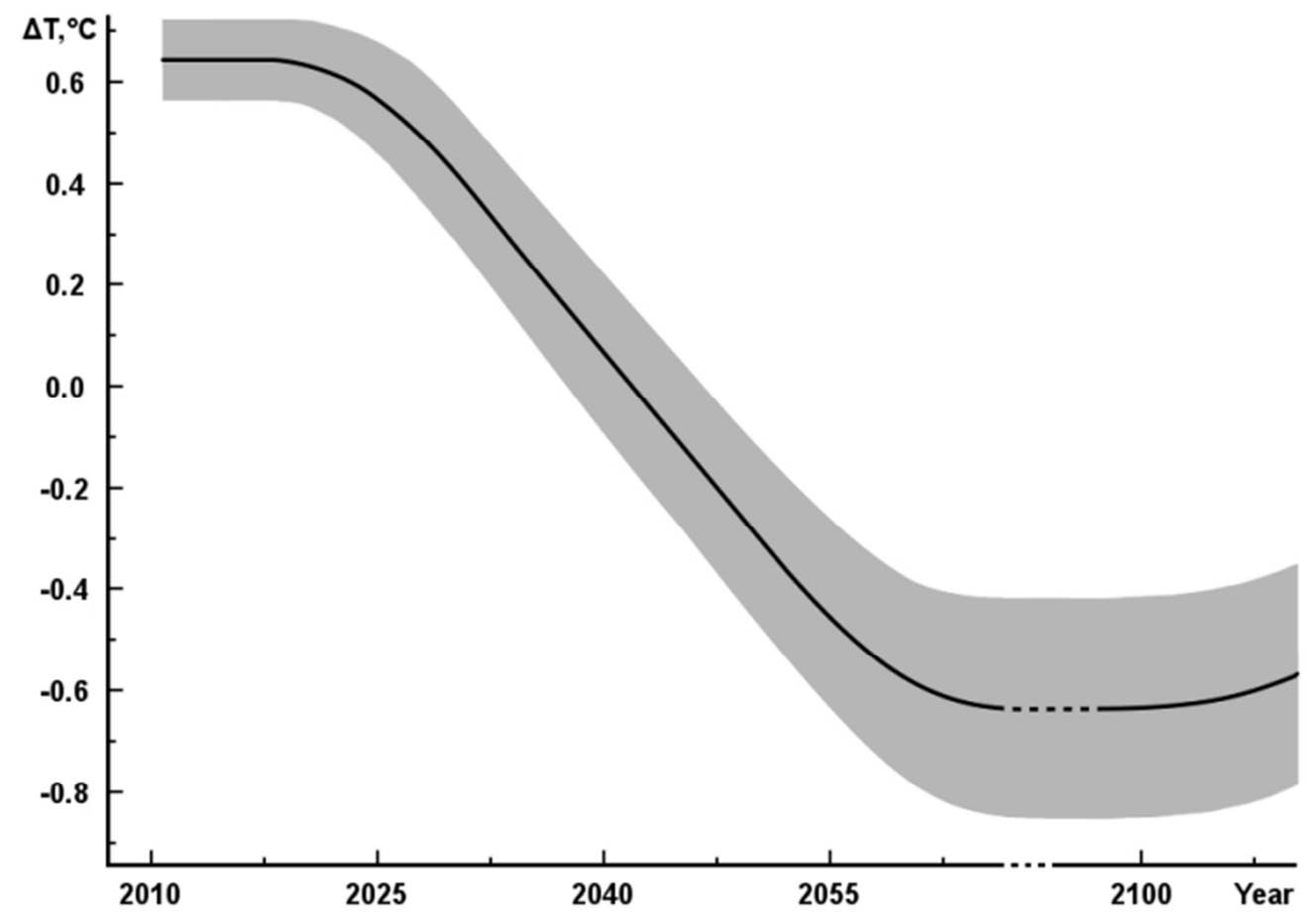

Figure 4. The forecast of temperature drops in the quasi-century era of the next 19th Little Ice Age over the past 7,500 years [3, 4].

1. an increase in the area of snow-ice covers and also changes in the physical properties (parameters) of the Earth's surface and atmosphere, and as a result, a significant increase in the Earth's Bond albedo;

2. reducing the concentration of the main GHG - water vapor and other GHGs in the atmosphere in accordance with the Clapeyron-Clausius ratio and Henry's law;

3. enhanced atmospheric transmission of thermal radiation of the Earth's surface ria the transparency window;

4. reducing the "dark" surface of Ocean, caused by a decrease in the water level of the oceans due to cooling and the corresponding heat compression of water (during cooling).

Such additional temperature decreases, in turn, lead to the following turns (cycles) of intensification of cooling. A long chain of such cycles can up to several times the impact of TSI to climate and lead to a deep cooling up to Little Ice Age. Consequently, quasi-bicentennial cyclic variations of TSI (up to $\sim 0.4 \%$ according to Shapiro et al. [5] and Egorova et al. [6]) together with very important followed of a long chain by multiple influences of the secondary causal feedback effects determine the appropriate climate changes from warming up to Little Ice Age [2, 3, 4, 18, 19]. The temperature is always cooler (with some delay) during longterm periods of low TSI of recession phase and warmer during periods of high TSI of growth phase is an important aspect of the climate impact of TSI quasi-bicentennial cycle. The history of the climate of our planet is a chain of quasi- 
bicentennial continuously alternating cycles due to the corresponding variations of TSI. So, the climate varies with the TSI quasi-bicentennial cycle. Therefore, a long-term average annual energy imbalance between the Earth and space is of fundamental importance to the climate system. In so doing a long-term representative data on the global energy of the climate system are extremely important.

\section{Absence of the Impact of the Flux of Cosmic Rays and the Cloud Coverage on the Energy Balance of the Earth}

The energy of solar radiation absorbed by the Earth, as well as fraction of the thermal radiation of the Earth's surface, which is released to the space through the atmospheric transparency window, generally depends on variations of the area of the cloud cover. Svensmark et al. $[20,21,22,23]$ suggest that the increase in the area of the cloud cover in the lower atmosphere, presumably caused by an increase in the flux of galactic cosmic rays during the Grand quasi-bicentennial minimum of solar activity results only in an increase reflected fraction of the solar radiation to space and weakens the flux of the solar radiation that reaching Earth surface. Without any corresponding calculations of the variations of the average annual energy balance of the Earth $E$ they suggested that the consequences will include only a deficit of the solar energy absorbed by the Earth and a cooling of the climate up to the onset of the Little Ice Age. These suggestions ignore simultaneous impact of the opposite aspects of the increase in the area of the cloud cover on the climate warming. The latter will result from a decrease in the power of thermal radiation of the Earth's surface released to the space, and also in the power of the solar radiation reflected from the Earth's surface, due to the increase in their absorption and reflection back to the surface by increased clouds coverage. A substantial strengthening in the greenhouse effect and the narrowing of the atmospheric transparency window will also occur.

Abdussamatov [24, 25] impact assessment was carried out of all aspects of possible long-term $2 \%$ growth of the cloud cover area in the lower atmosphere on the average annual energy balance of the Earth $E$. An increase in the cloud cover area in the lower atmosphere will result simultaneously both in the decrease and in the increase in the temperature, which will virtually compensate each other, while the energy balance of the Earth $E$ before and after the increase in the cloud coverage by $2 \%$ will stay essentially the same: $E_{1}-E_{\mathrm{o}}$ $\approx 0$. An impact of the long-term growth in the cloud coverage (presumably caused by an influence of an increase in the cosmic ray flux) on variations in the annual average energy budget of the Earth and, consequently, on the climate is virtually nonexistent. Thereby, the Earth climate does not depend on variations of cosmic rays and the cloud cover and is determined only by long-term variations in energy imbalances between the Earth and the space, as well as by impacts subsequent numerous feedback effects.

\section{A Water Vapor Rise Makes the Temperature Less Sensitive to the Atmospheric Carbon Dioxide Increase}

The natural abundance of water vapor and carbon dioxide in the atmosphere, in accordance with the ClapeyronClausius relation and the Henry's law, varies significantly depending on temperature changes, increasing to a maximum level during warming and dropping to a minimum level in the Ice Ages (additionally significantly enhancing and weakening accordingly the influence of the GHG effect). However, due to the substantial overlap of the absorption spectral bands of water vapor and carbon dioxide with each other in the ranges of $\sim 4-5, \sim 9-11$, and $\sim 12-18 \mu \mathrm{m}$ (Figure 5) the absorption of thermal radiation of the Earth's surface with increasing temperature does not occur in proportion to the increase in their absolute concentrations in the atmosphere. Since, according to the vertical profiles of volumetric concentrations of water vapor and carbon dioxide in the atmosphere, even a slight simultaneous increase in their average concentration during warming leads to a sharp increase in the concentration of water vapor in the nearsurface layer with increasing of a concentration of the carbon dioxide, uniformly mixed in height (Figure 6). This is determined by the fact that almost all water vapor of the atmosphere, in contrast to carbon dioxide uniformly mixed to heights up to $60 \mathrm{~km}$, is concentrated in the troposphere and has a maximum concentration directly in the near-surface layer, and very sharply decreasing with height in the troposphere.

A long-term increase in TSI in the growth phase of the quasi-bicentennial cycle with a delay of $30 \pm 10$ years, determined by the thermal inertia of the Ocean, leads to an increase in temperature (Figure 3). This leads to an increase in the concentration of both the water vapor and carbon dioxide in the atmosphere. Even a slight increase in the average water vapor concentration leads to a multiple increase in its content in the near-surface layer. As a result, transfer of the surface radiations varies appreciably, since the spectral absorption bands of the water vapor and carbon dioxide overlap. A sharp increase directly in the atmospheric near-surface layer of the ratio of the density of volumetric concentrations of water vapor to carbon dioxide during warming leads to a relative increase in the absorption fraction by water vapor the surface thermal radiation in the overlapping spectral bands of the atmospheric transparency window. Variations in absorption spectrum corresponding to rise in the total water vapor concentration in the atmosphere on $7 \%$ and carbon dioxide since 350 until 420 ppmv during warming were simulated with fixed cloudiness. In so doing the fraction of surface thermal radiation absorbed by carbon dioxide in the overlapping sections of the spectral absorption bands of the atmospheric transparency window practical not increase due to increased absorption by sharply increased water vapor molecules directly in the lowest near-surface layers of the troposphere. As a result, even a slight increase 
in the total concentration of water vapor in the atmosphere reduces (neutralizes) the overall effect of the absolute increase in the concentration of carbon dioxide in the entire atmosphere on the thermal regime of the planet, i.e. the sensitivity of climate to the carbon dioxide abundance increase (at its current high level) decreases as a result of an even more significant increase in the concentration of water vapor directly in the near-surface layer of the troposphere during warming.

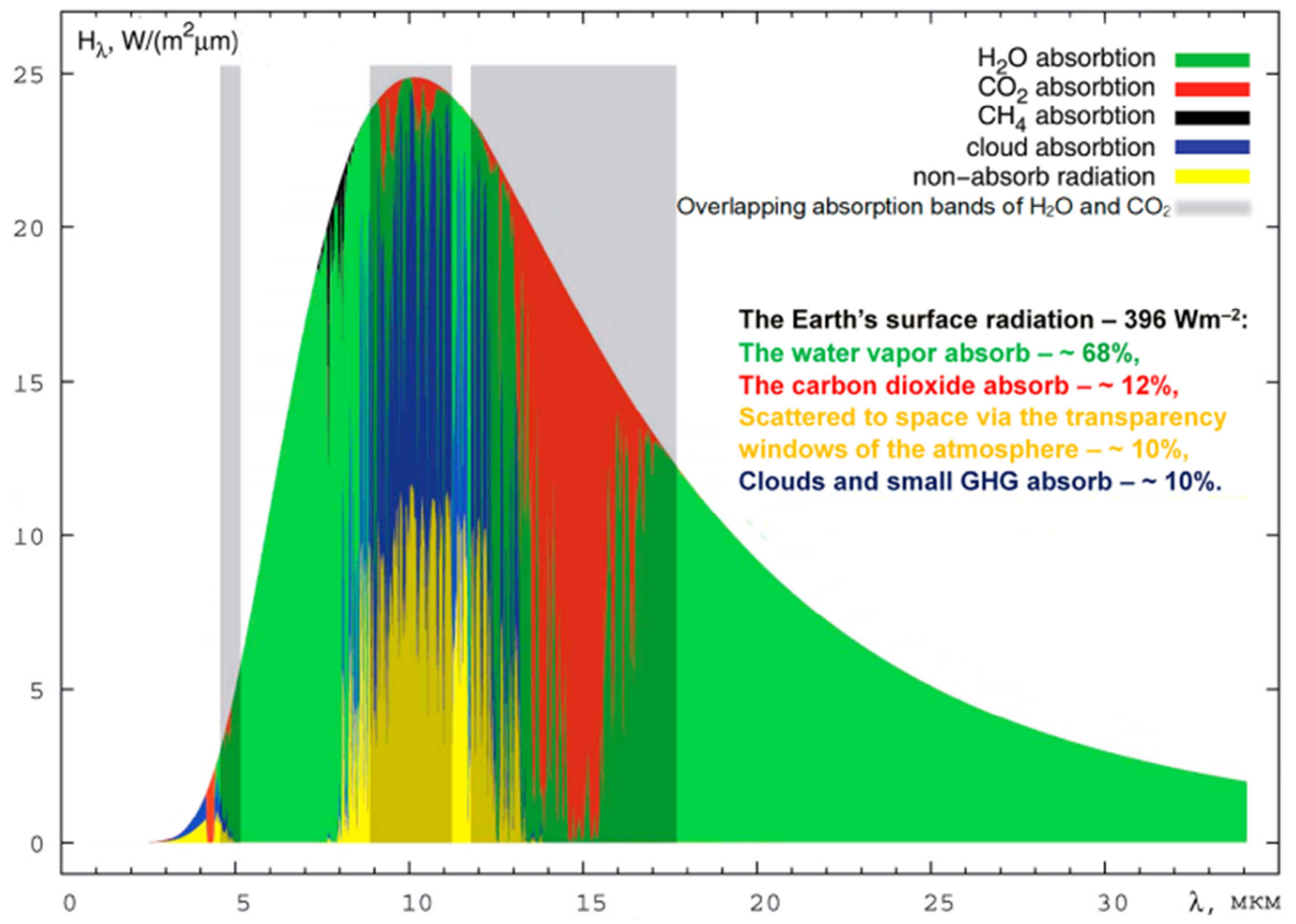

Figure 5. Spectral density of the thermal long-wave radiation flux from the surface (as a blackbody) of the Earth [3, 4].

In the wavelength ranges of the atmospheric transparency windows, carbon dioxide, having very weak absorption bands, will practically not be able to further significantly increase the absorption of thermal radiation of the surface due to the simultaneous parallel sharp increase in the concentration of water vapor during warming in the nearsurface layer of the atmosphere. Therefore, with an increase

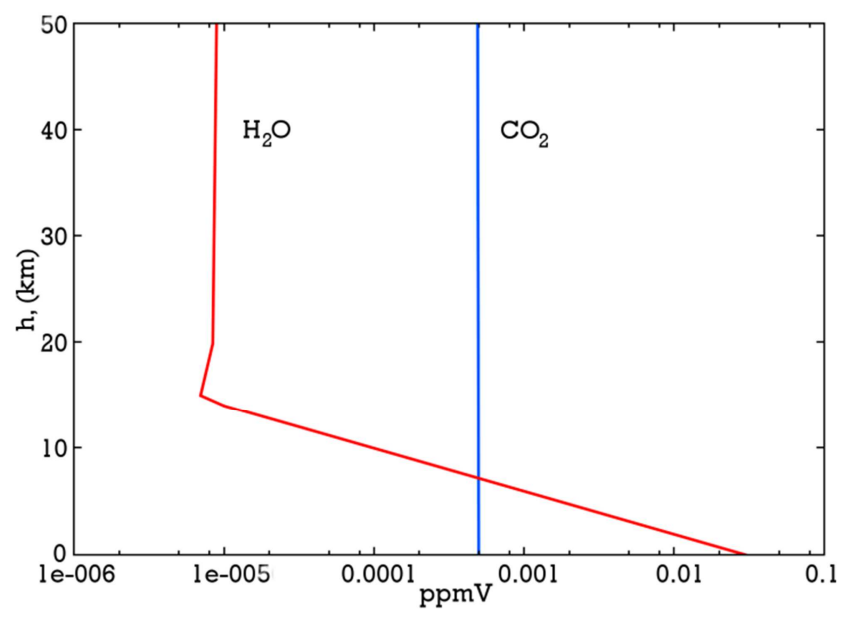

Figure 6. The changes in the concentrations of water vapor and carbon dioxide with height $h$ in the atmosphere [4]. in the concentration of carbon dioxide in the atmosphere (at its current high level of more than $415 \mathrm{ppmv}$ ), it is practically impossible to expect a significant additional increase them absorption of the surface thermal radiation. The impact of carbon dioxide on climate change is secondary and negligible compared to the effects of the long-term variation the total solar energy and abundance water vapor, i.e. the quasibicentennial variations of the solar irradiance reaching Earth is the main driver variations temperature of the surface, and not vice versa - variations concentration of carbon dioxide. Since "the amount of natural flows of carbon dioxide and water vapor from the oceans and land to the atmosphere $\left(\mathrm{M}_{\mathrm{in}}\right)$ and from the atmosphere $\left(\mathrm{M}_{\text {out }}\right)$ to the oceans and land is many times greater than the anthropogenic emissions of these substances into the atmosphere $\left(\mathrm{M}_{\text {ant }}\right)$. The total carbon dioxide content in the oceans is about 50 times higher than in the atmosphere" $[4,26]$. With a slight increase in the water temperature the amount of natural carbon dioxide transferred from the Ocean to the atmosphere will become significantly larger than carbon dioxide absorbed by the Ocean from the atmosphere. The remaining surplus of carbon dioxide in the atmosphere exceeds its growth based on the growth of its emissions from human activity. 


\section{Conclusions}

Study of significant climate fluctuations during the last 800,000 years show that quasi-bicentennial and one hundredthousand-year cyclic variation the TSI are the main reasons of alteration of warming and cooling before the Little Ice Age and Grand Ice Ages. Changes in parameters of the Earth's surface and atmosphere (the Earth's Bond albedo, the concentration of water vapor and others GHGs in the atmosphere, etc.), caused by cooling (warming), will generate a long chain of an appropriate effect significant additional influence. The observed parallel warming in the last quarter of the twentieth century on Mars, Jupiter, Triton, Pluto and others confirm that in the SS also by analogy with the earthly seasons there is a quasi-bicentennial alternation of climatic conditions [27, 28]. From this point of view, the "sunny summer" season ended in the SS and the "sunny autumn" season began, and then approximately in the year $2070 \pm 11$ the "sunny winter" season will begin. "Spring" in the SS will come only at the beginning of the XXII century $[3,4,10]$. The Sun is the main factor controlling the Earth's climate system, and even minor long-term changes in the power of its radiation can have serious consequences for the Earth's climate. A long-term monitoring deviation of the energy balance of the planet from the equilibrium condition will allows to determine the value accumulated in the Ocean excess of the incoming solar energy (with positive sign) or magnitude of the accumulated deficiency (with negative sign). Therefore, it defines the direction and depth of the future climate changes into account thermal the inertia of the Ocean - atmosphere system and the forecast of the upcoming variation of the TSI quasi-bicentennial cycle. Natural quasibicentennial changes in the Earth's energy balance remain the main factor in climate change in the past, present and near future, and the cause of the onset of Little Ice Age is the Grand and long deep TSI minimum of the quasi-bicentennial cycle. The quasi-bicentennial cyclical variation of TSI, together with the significant impact of a long chain of subsequent causal feedback effects, governs and completely determines the alternation of climate change from warming to Little Ice Age and is the main factor controlling and managing the climate system. At the same time, cyclical variations in solar activity, which correlated with climate change, are the accompanying phenomena of physical processes occurring in the interior of the Sun and do not substantially affect either the TSI and the Earth's climate. Since the 11-year and quasi-bicentennial cyclic variations in solar activity and TSI, being a manifestation of the same processes in the solar interior, are interconnected and synchronized in both phase and amplitude.

The energy of solar radiation absorbed by the Earth, as well as the thermal radiation of the Earth's surface, which is released to the space through the atmospheric transparency window, generally depends on variations of the area of the cloud cover. However, an increase in the cloud cover area in the lower atmosphere will result simultaneously both in the decrease and in the increase in the temperature, which will virtually compensate each other, while the energy balance of the Earth $\mathrm{E}$ before and after the increase in the cloud cover area by $2 \%$ will stay essentially the same: $E_{1}-E_{\mathrm{o}} \approx 0$. An impact of the long-term growth in the cloud coverage (presumably caused by an influence of an increase in the cosmic ray flux) on variations in the annual average energy budget of the Earth and, consequently, on the climate is virtually nonexistent. The Earth's climate does not depend on variations in cosmic rays and cloud coverage; it is defined solely by the long-term change in the energy imbalance between the Earth and outer space, as well as multiple subsequent feedback effects [24, 25].

Increase in TSI in the growth phase of the quasibicentennial cycle with a delay of $30 \pm 10$ years [17], determined by the thermal inertia of the world ocean, leads to an increase in temperature (Figure 3), which leads to an increase in the concentration of both the water vapor and carbon dioxide in the atmosphere. In so doing due to the significant overlap of the spectral absorption bands of water vapor and carbon dioxide, the climate sensitivity to the atmospheric carbon dioxide abundance decreases as a result of a significant increase in the concentration of water vapor directly in the near-surface layer of the troposphere during warming. As a result, even a slight increase in the total concentration of water vapor in the atmosphere during warming reduces (neutralizes) the overall effect of the corresponding increase of the absolute abundance of carbon dioxide in the entire atmosphere on the thermal regime of the planet. Therefore, with an increase in the concentration of carbon dioxide in the atmosphere (at its current high level of more than $415 \mathrm{ppmv}$ ), it is practically impossible to expect a significant additional increase in absorption by them of thermal radiation of the surface. The impact of carbon dioxide on climate change is secondary and negligible compared to the effects of a long-term variation in the atmospheric water vapor concentration, the Earth's Bond albedo and the total solar energy of the quasi-bicentennial cycle reaching the Earth.

\section{Acknowledgements}

This study was partly supported by the Project No. KP19270 of the Praesidium of the Russian Academy of Sciences for Fundamental Scientific Research ("Issues of the Origin and Evolution of the Universe Using Methods of Ground-Based Observations and Space Research"). I express my gratitude to Dr. A. A. Mushtukov for his help in preliminary conducting an estimated simulate of the variations in the absorption spectrum of the water vapor and carbon dioxide at a small increase they abundance in the atmosphere during warming.

\section{References}

[1] Abdussamatov, H. I. (2005). Long-term variations of the integral radiation flux and possible temperature changes in the solar core // Kinematics and Phys. Celest. Bodies. 21, 328332. 
[2] Abdussamatov, H. I. (2012). Bicentennial decrease of the solar constant leads to the Earth's unbalanced heat budget and deep climate cooling. Kinematics and Phys. Celest. Bodies. 2, 6268 .

[3] Abdussamatov, H. I. (2015). Current long-term negative average annual energy balance of the Earth leads to the new Little Ice Age. Thermal Sci. 19, S279-S288.

[4] Abdussamatov, H. I. (2016). The new Little Ice Age has started. Evidence-Based Climate Science. Easterbrook D. J. (ed) Oxford: Elsevier, 307-328.

[5] Shapiro, A. I., Schmutz, W., Rozanov, E., Schoell, M., Haberreiter, M., Shapiro, A. V., Nyeki, S. E. (2011). A new approach to the long-term reconstruction of the solar irradiance leads to large historical solar forcing. Astron. Astrophys. 529, A67.

[6] Egorova, T., Schmutz, W., Rozanov, E., Shapiro, A. I., Usoskin, I., Beer, J., Tagirov, R. V., Peter, T. (2018). Revised historical solar irradiance forcing. Astron. Astrophys. 615, A85.

[7] Fröhlich, C. (2016). Solar Constant www.pmodwrc.ch/pmod.php?topic=tsi/composite/SolarConsta nt.

[8] SunSpot Data. (2014). SIDC-Solar Influences Data Analysis Center, http://sidc.oma.be/sunspot-data/.

[9] Milankovitch, M. (1998). Kanon der erdbestrahlungen und seine anwendung auf das eiszeitenproblem. In: Canon of insolation and the Ice Age problem. With Introduction and biographical essay by Nikola Pantic. Hardbound Alven Global Belgrade, 636 pp (in English).

[10] Abdussamatov, H. I. (2017). Lunnaya observatoriya dlya issledovanii klimata Zemli $\mathrm{v}$ epokhu glubokogo pokholodaniya (Lunar Observatory for Earth climate studies in the deep Ice Age) St. Petersburg: Nauka, 2017.

[11] Abdussamatov, H. I. (2006). The time of the end of the current solar cycle and the relationship between duration of 11-year cycles and secular cycle phase. Kinematics and Phys. Celest. Bodies. 22, 141-143.

[12] Abdussamatov, H. I. (2015). Power of the Energy of 11-Year Solar Cycle and Its Dependence on Solar Cycle Length. Kinematics and Phys. Celest. Bodies. 31, 54-60.

[13] Climate Change: New Antarctic Ice Core Data. 2000. http://www.daviesand.com/Choices/Precautionary Planning/N ew_Data/.

[14] Fischer, H., Wahlen, M., Smith, J., Mastroianni, D., Deck, B. (1999). Ice core records of atmospheric $\mathrm{CO}_{2}$ around the last three glacial terminations. Science. 283, 1712-1714.

[15] Pedro, J. B., Rasmussen, S. O., van Ommen, T. D. (2012). Tightened constraints on the time-lag between Antarctic temperature and $\mathrm{CO}_{2}$ during the last deglaciation. Climate of the Past. 8, 1213-1221.

[16] Petit, J. R., Jouzel, J., Raynaud, D., Barkov, N. I., Barnola, J.-
M., Basile, I., Bender, M., Chappellaz, J., Davis, M., Delaygue, G., Delmotte, M., Kotlyakov, V. M., Legrand, M., Lipenkov, V. Y., Lorius, C., PÉpin, L., Ritz, C., Saltzman, E., Stievenard, M. (1999). Climate and atmospheric history of the past 420,000 years from the Vostok ice core, Antarctica. Nature. 399, 429-436.

[17] Abdussamatov, H. I., Bogoyavlenskii, A. I., Khankov, S. I., Lapovok, E. V. (2010). Modeling of the Earth's planetary heat balance with electrical circuit analogy. Journal of Electromagnetic Analysis and Applications. 2, 133-138.

[18] Abdussamatov, H. I. (2019). Climate change under the influence of the Sun in present and future. Problems of Geography. N 149. 220-262, (in Russian).

[19] Nils-Axel, M. (2015). The approaching new Grand solar minimum and Little Ice Age climate conditions. Nat. Sci. 7, $510-518$.

[20] Svensmark, H., Friis-Christensen, E. (1997). Variation of cosmic ray flux and global cloud coverage - a missing link in solar-climate relationships. J. Atmos. Sol.-Terr. Phys. 59, 1225-1232. https://doi.org/10.1016/S1364-6826(97)00001-1.

[21] Svensmark, H. (2007). Cosmoclimatology: A new theory emerges. Astron. Geophys. 48, 1.18-1.24. https://doi.org/10.1111/j.1468-4004.2007.48118.x.

[22] Svensmark, H., Enghoff, M. B., Shaviv, N. J., Svensmark, J. (2017). Increased ionization supports growth of aerosols into cloud condensation nuclei. Nat. Commun. 8, 2199, https://doi.org/10.1038/s41467-017-02082-2.

[23] Stozhkov, Y. I., Bazilevskaya, G. A., Makhmutov, V. S., Svirzhevsky, N. S., Svirzhevskaya, A. K., Logachev, V. I., Okhlopkov V. P. (2017). Cosmic rays, solar activity, and changes in the Earth's climate. Bull. Russ. Acad. Sci.: Phys. $81,252-254$.

[24] Abdussamatov, H. I. (2018). Cosmic rays and clouds variations effect on the climate is insignificantly. Applied Physics Research. 10, 81-86.

[25] Abdussamatov, H. I. (2019). The Earth's climate does not depend on variations in cosmic rays and cloud coverage. Geomagnetism and Aeronomy. 59, 935-941.

[26] Nigmatulin, R. I. (2010). The ocean: climate, resources, and natural disasters. Herald of the Russian Academy of Sciences 80, 338-349.

[27] Odyssey studies changing weather and climate on Mars. (2005). The changing south polar cap of Mars: 1999-2005, MGS MOC Release no. MOC2-1151.

[28] Ravilious, K. (2016). Mars melt hints at solar, not human, cause for warming, scientist says. National Geographic News. May 6.

[29] Abdussamatov, H. I., Khankov, S. I., Lapovok, Ye. V. (2011). Factors defining the thermal inertia characteristics of the system Earth - atmosphere. Proceedings of the All-Russian annual conference on solar and solar-terrestrial physics. St. Petersburg. 307-310, (in Russian). 